\title{
Neural Bases of Sequence Processing in Action and Language
}

\author{
Francesca Carota and Angela Sirigu \\ CNRS
}

Real-time estimation of what we will do next is a crucial prerequisite of purposive behavior. During the planning of goal-oriented actions, for instance, the temporal and causal organization of upcoming subsequent moves needs to be predicted based on our knowledge of events. A forward computation of sequential structure is also essential for planning contiguous discourse segments and syntactic patterns in language. The neural encoding of sequential event knowledge and its domain dependency is a central issue in cognitive neuroscience. Converging evidence shows the involvement of a dedicated neural substrate, including the prefrontal cortex and Broca's area, in the representation and the processing of sequential event structure. After reviewing major representational models of sequential mechanisms in action and language, we discuss relevant neuropsychological and neuroimaging findings on the temporal organization of sequencing and sequence processing in both domains, suggesting that sequential event knowledge may be modularly organized through prefrontal and frontal subregions.

\section{Introduction}

The ability to estimate in real-time what will come next when we start acting is a crucial prerequisite for planning sets of coherent behavioral units. For action plans to result in optimally purposive patterns of concatenated individual moves, the specific temporal structure associated to the required sequence of actions needs to be computed prospectively based on adequate representations of event knowledge.

The psychological categorization of the causal-event structure that is inherent to human knowledge has been a vexata crux in the field of cognitive psychology, because sequential structures permeate conceptually distinct knowledge domains.

In action, for instance, sequential structures affect movement selection and prediction during the formulation of motor programs and higher order,

Correspondence concerning this article should be addressed to Francesca Carota, Centre de Neuroscience Cognitive, 67 Blv Pinel, 69675 Bron, France. Internet: Francesca.Carota@isc.cnrs.fr 
macrolevel action plans, as well as the analysis of observed series of action clusters. Accordingly, a degraded representation of such high-level structures of sequential knowledge has a direct impact on the physical ability to program motor commands, to control actions, and to achieve its related goals, leading to incomplete or purposeless behaviors (Shallice, 1988; Sirigu, Zalla, Pillon, Grafman, Dubois, et al. 1995; Sirigu et al. 1996, 1998; Zalla, Plassiart, Pillon, \& Sirigu, 2001; Zalla, Pradat-Diehl, \& Sirigu, 2003).

Sequential information is also a crucial cognitive component in an a priori distinct domain such as language, which exhibits sequential patterns at multiple structural dimensions, such as morphology and syntax. For instance, both local and long-distance syntactic dependencies in natural language (e.g., topicalization, wh-movement, etc.), with their embedded semantic relations, may require computational procedures and a working memory system parallel to the ones that are involved in action sequencing. It has been proposed, for instance, that the syntactic component of the language faculty may derive from a set of prelinguistic operations borrowed from the motor planning domain (Steedman, 2002). On the other hand, language may be treated within different temporal spans due to different constraints of memory load and domain-specific governing principles. This would be in line with classical dual-system views of language (Chomsky, 1995, 1998; Pinker, 1994; Pinker \& Ullmann, 2002; Ullmann 2001, 2004), which posit that the mental grammar employs an autonomous set of procedural rules capturing regularities intrinsic to language and specifying possible hierarchical and sequential combinations between morphological and lexical material (stored in declarative memory) into richer representations, such as complex words and sentences.

The relevance of sequential information in action and language leads to the question of how these related domain-specific categories of sequential knowledge are internally represented.

Sequential event knowledge is commonly assumed to be stored based on memorized sequence configurations, the so-called structured event complexes (Grafman, 1995, 1999; Wood \& Grafman, 2003), also known in terms of schemas (Bartlett, 1932) and scripts (Schank \& Abelson, 1975). These internal representations of goal-oriented sets of subsequent cognitive representations of events specify at a high degree of unification the global order of event series, encompassing chronological, linguistic, and motor information (Wood et al., 2004).

In this perspective, it becomes particularly interesting to address the specific question of whether the categorization, activation, and processing of different 
event knowledge domains rely on a common neural processor or, rather, on a domain-dependent one.

It is widely acknowledged that the prefrontal cortex plays a central role in the storage, the maintenance of sequence representation, and the generation of sequential processes. However, whether and how diverse knowledge categories are reflected and subserved by specialized or overlapping neural codes within the prefrontal regions has been a matter of long-standing scientific debate. Ample work suggests that the specific functional organization of this brain area is largely sensitive to different, domain-specific aspects of event knowledge. A possible explanation of such specialized subregions would be the connectivity with posterior cortical and/or subcortical regions whose functions are related, for example, to higher order sensory processing (parietal cortex, association areas), memory (hippocampus), and motor control (premotor cortex, supplementary motor area, basal ganglia) (Wood \& Grafman, 2003). The particular cytoarchitectonic structure (i.e., the arrangement and density of nerve cells in the cerebral cortex; from Greek $\kappa \dot{v} \tau o \varsigma=$ cell $+\alpha \rho \chi \iota \tau \varepsilon \kappa \tau o \nu \iota \kappa \dot{\eta}=$ architecture) and connectivity of the region (i.e., the connections of its nervous fibres with noncontiguous brain regions) motivates the interaction between the sequential event knowledge system and the neural bases of temporal processing, memory, action, and language.

The present article attempts to characterize the representations of sequential event knowledge structures and the functions of the prefrontal cortex in mediating the computational operations required to generate and process them in action and language.

The remainder of the article is structured as follows. First, some representative models of sequential event structures are sketched from the perspective of cognitive psychology and linguistic theory. This prepares the ground for introducing, as a second concern, the neuroanatomical and functional correlates of sequence processing, focusing on relevant findings from neuropsychological and neuroimaging work for particular dissociations between different categories of event knowledge represented by action and language sequences. We then conclude by profiling the implications of these results for the current models of sequential knowledge processing.

\section{Sequential Event Structures}

The representation of sequential event structures is based on a temporal and causal ontology that is rooted in our cognitive ability to perceive and discriminate between two types of events: (a) event types that involve continuous update 
and change over time, namely processes, and (b) event types that do not temporally change, namely states (Langacker, 1987, p. 258). This ability allows us to establish the relative order between contiguous events in terms of initial events and subsequent ones, which is a prerequisite for planning and processing strategies both in action and language.

In the present article we focus on the particular type of temporal processing of information that is required in sequential structuring and that allows us to establish the relative order of the events in terms of precedence and dominance relations between them.

The ontological dichotomy between states and processes is especially mirrored in verbal Aktionsart, a part of the aspectual system, but it is also somehow reflected by the representational models that have been developed within cognitive psychology, some of which include a dynamic principle, whereas others emphasize the static organisation of data structures, as we will argue in the next section.

\section{Frames, Chunks, and Scripts}

Multidisciplinary approaches have accounted for the modelling of event sequences in terms of structured templates. We will briefly introduce three major representational models, a meta-scheme independent of the specific domain such as frame, an action-related one, that is, a chunk, and a language-related one, that is, a script.

The attempt to unify the structuring of reasoning, memory, and language under a common abstract denominator inspired Minsky (1975) to propose that minimal knowledge units, or frames, interface with the respective "factual and procedural contents" of these domains. Defined as a remembered framework to be adapted to fit reality by changing details if necessary, a frame provides a basis for effective adaptative responses to novel situations. However, it consists of a rather static "data structure for representing a stereotypical situation" (Minsky, p. 212), which specifies several types of information, such as instructions about the use of the frame itself, expectations about upcoming events, and steps to undertake when expectations are disconfirmed. For our purpose, one relevant aspect of the frame notion is that it is formally modeled as a treelike network of nodes and relations, which are hierarchically related, with fixed root nodes for true properties of known situations and terminal leaves to be filled by specific instances depending on particular restrictions to be met for satisfying a slot-filler assignment condition. As a result, multiple frames can become interrelated in complex systems, by means of multiple combinations of event structures. 
Likewise, event knowledge representation specific to goal-directed action has been modeled in the form of memorized representation units, or chunks (Black \& Bower, 1979). The process of chunking information, which refers to grouping or clustering together the discrete information units called chunks, is usually assumed to facilitate the understanding and the retrieval of knowledge representations in human short-term memory (Miller, 1956). The psychological reality of chunk-based representations has been specified in terms of episodes containing structures of embedded goals and subgoals (Black \& Bower). The particular structure of chunks has an effect on memory. In fact, the chunks contain hierarchically interconnected events, embedded according to different levels of abstraction, including basic, subordinate, and superordinate goaldirected events. The basic events consist of the sensorimotor and physical features involved in the performed actions, whereas the events of higher levels are related to the long-term goal of actions. The basic events, such as motor representations, are processed automatically by perceptual chunking. On the other hand, superordinate events that require intentional control of the related goals are encoded, processed, and recalled on the basis of a goal-oriented chunking principle (Gobet et al., 2001). The hierarchical organization of the goal-oriented events has a direct impact on recall performance, because superordinate events are better recalled when more subordinate events are encompassed within the same episode and inferred from the corresponding superordinate ones.

The interest of the chunk-based model is the conceptual transition from sequential event knowledge representations to goal-oriented action, which also underlies the notion of script, a structure that configures an appropriate sequence of events in a particular context, relying on slot fillers, or attributevalue associations, in which each event is interrelated with the subsequent one forming a whole (Schank \& Abelson, 1975). Sequences are said to be internally constrained by a causal chaining principle (Abelson, 1973; Schank, 1973), imposing co-occurrence restrictions on sequence units and script sequences according to a "what-if" alternative that allows rejection of incoherent units and reduces order error outputs. Importantly, the script framework extends knowledge representations to event plans. In fact, a parameter of goal-directedness marks the difference between scripts themselves and the set of event sequences (i.e., plans) that are oriented toward a goal, based on a set of intention-driven choices. Accordingly, the internal organisation of scripts and plans is an arborescence built on the causal relationships between event representations, goals and subgoals, respectively.

In the perspective of scriptlike knowledge structure, texts exemplify series of events correlated with goals and plans necessary to achieve them: The 
stories they report offer stylized representations of scripts, and text structure is designed as a set of paths joined at crucial points, temporally and hierarchically ordered.

The models just sketched share a hierarchical ordering of events, cooccurrence constraints on allowed subsequent events, and goal orientation of plans. These features of sequential event knowledge representation are valid not only for the action domain but also for language, as we will briefly consider while discussing their impact on narrative discourse macrostructures and syntactic microstructures.

\section{Macrostructures: Narrative Discourse}

It has been claimed that the representational devices that organize world knowledge for predicting and interpreting new situations, information, and events of experience are "structures of expectations" (Tannen, 1979), which can be applied to language. Language in action, or talk, is indeed shaped through a series of contextually dependent "shifting frames" caused by mismatches in schemas, or expectations about events, objects, and individuals (Tannen \& Wallat, 1987). Schank (1973), in his early work on natural language, described how a word in a single sentence triggers expectations about what will follow in the rest of the sentence, and a single sentence about what will follow in the rest of the discourse story, pointing out that the whole of these expectations form world knowledge related to situations.

A caveat regarding extending the notion of expectation toward an intentional goal to language comes from theories of discourse that posit a distinction between linguistic and nonlinguistic discourse dimensions. For instance, the linguistic structure of the actual sequence of utterances in discourse is distinct from the intentional structure that is determined by the goals underlying speakers' communicative actions and from the attentional state, or focus of attention recording and keeping track of the referents, the properties, and relations between discourse segments that are salient at each stage of the unfolding discourse (Grosz \& Sidner, 1986). Accordingly, although discourse linguistic structure can be segmented, horizontally, into segments and embedded segments by detecting the segment boundaries, it is the intentional dimension that specifies both precedence relations and dominance relations between discourse segments, determining their hierarchical order in terms of planned goals and subgoals. To process discourse thus means to recognize how the utterances of the discourse combine into segments, as well as to understand the underlying intentions and the relationships of precedence and dominance among them. It also means to construct and organize a mental model (i.e., an "attentional 
state" [or focus of attention] that records, tracks, and dynamically updates behavior).

A question is thus whether, and to what extent, during narrative discourse processing and planning these recognition tasks depend on features that are merely textual (or internal to the linguistic dimension) or also depend on the speakers' knowledge of the discourse domain.

A pertinent point that emerges from psycholinguistic findings is that the planning of the causal structure encompassing orderly causes and consequences is indeed based on fine-grained textual, linguistic features inherent to discourse, such as the contextual accessibility and recency of lexical anchors, or antecedents, of anaphoric expressions; that is, speakers decide what to say next based on (co)text or prior discourse. Interestingly, however, the temporal structure of ongoing narrative also determines the selection procedure leading speakers to choose what they will say next with respect to temporally prior discourse (Simner \& Pickering, 2004).

This tells us that, in narrative discourse, the planning of the causal structure of a sequence of consecutive causes and consequences is driven by both linguistic properties and temporal information about the typicality of events and, ultimately, event knowledge.

Although a language-specific component is preserved from the intentional structure underlying communicative actions in language, narrative discourse becomes representative of plans, or goal-directed sequential structures, in both language and action domains. For this reason, it has offered an exclusive test bed for theories and hypotheses about the domain sensitivity of the organization of event knowledge.

It remains now to consider whether a similar temporal treatment also applies to microstructural, local language sequences, such as syntactic structures.

\section{Microstructures: Syntax}

Syntax specifies grammatical functions between word constituents to encode hierarchical syntactic relationships, precedence relations in word order, and inflection. Processing and parsing syntactic structure imply the analysis and the decomposition of syntactic constituents by recognizing the relations of immediate dominance and linear precedence between them. For example, in the generative tradition, syntax has been configured as a sequence of structure-building operations such as Merge, or concatenation, a one-step, recursive computational procedure that captures generalized transformations to combine autonomous syntactic trees under a single node (Chomsky, 1993, 1995). 
Recent theoretical views attribute great centrality to this type of operations, such as merging or unification, which allow the combination of language material into sequential patterns by discarding unselected competitor candidates, as being a powerful computational mechanism in grammar (Hagoort, 2005; Jackendoff, 2002).

Interestingly, work on combinatory categorical grammar has recently extended a similar view to the action domain. More specifically, a recent hypothesis suggests that composition, a procedure that combines representations and event functions into sequences, constitutes an explicit formal parameter interfacing syntax and simple planned action: In both domains, the causal association between initial, preparatory events and consequent, future outcomes originates in the process of planning, and the related notion of goal-directedness (Steedman, 2002).

It is known that the need to plan and hierarchically combine linguistic units arises because of the mismatch between conceptual messages and word sequences that are linear and spread over time (Levelt, 1989). This process requires the assignment of a relative order between elements, including both motor actions at the base of articulated speech, and syntactic constituents. What it ultimately presupposes is thus the coordination of this assignment mechanism with the planning of the motor unit sequences that serve to package and structure the information flow. In this perspective, the planning and the organization of both motor action and syntactic sequences are closely interrelated in language.

We can now turn to the neuroscientific issue of how sequential event knowledge structures are encoded and processed in the brain. As previously mentioned (see introduction), the question we intend to address is whether specialized neural substrates represent and separately treat sequences of events belonging to specific domains of knowledge, such as action or language. Alternatively, the representation of sequential event structures in both domains could rely on a common neuroanatomical base.

In the next section, we attempt to give an answer by reviewing and discussing the ample literature dealing with the issue of the temporal organization of serially ordered cognitive events across dedicated cerebral networks.

\section{Sequence Processing in the Prefrontal Cortex}

Different representational and process-based models have been proposed to explain the role of the prefrontal cortex in action planning and monitoring (Wood \& Grafman, 2003). Nevertheless, there is general consensus that this brain region is a preferred location for the abstract representation and expression 


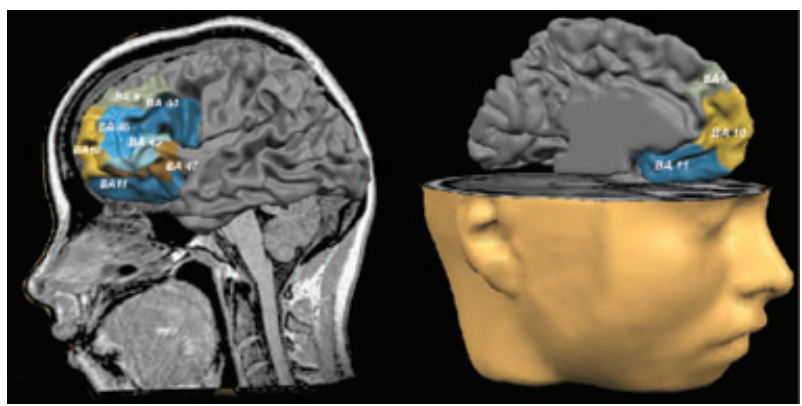

Figure 1 From left to right, lateral and medial views of the prefrontal regions. These include the medial prefrontal cortex (BA 11), the medial (BA 10) and lateral orbitofrontal cortex (BA 47), the dorsolateral prefrontal cortex (BA 9, BA 46), the pars triangularis (BA 45), and the pars opercularis (BA 44) of Broca's area. (All images were made in BrainVoyager; http://www.brainvoyager.com.)

of the temporal structure of complex motor action sequences. It has been widely suggested in the literature that the prefrontal regions play a functional role in the integration of temporally separate events into purposeful action sequences, and in the mediation of domain-specific representations of sequential event knowledge (Fuster, 1989; Goldman-Rakic, 1987; Grafman, 1989).

In fact, several studies provide converging evidence for a preferential involvement of prefrontal subregions in modulating various temporal aspects of sequential event knowledge (Crozier et al., 1999; Knutson, Westdorp, Kaiser, \& Hommer, 2000; Zacks Tversky, \& Iyer, 2001). For instance, positron-emission tomogarphy results show that the expression of emotional and nonemotional plans recruits the medial prefrontal and the dorsolateral prefrontal cortex, respectively (Partiot, Grafman, Sadato, Wachs, \& Hallett, 1995; cf. Figure 1). A particular network - with the inclusion of the right frontal lobe, left superior temporal gyrus, and the middle temporal gyrus bilaterally (Figure 2) - is specifically activated during the temporal ordering of script events, whereas a distinct circuit, more lateralized on the left hemisphere and including the left frontal lobe, left anterior cingulate, and the anterior part of the left superior temporal gyrus (Figure 2), is implicated in assigning an event to a given script or action category (Partiot, Grafman, Sadato, Flitman, \& Wild, 1996).

Neuropsychological studies demonstrate that a lesion in the prefrontal cortex is responsible for selective impairment in event sequence processing (Grafman, 1989): prefrontal patients fail to generate the correct temporal order of action sequences, in completing a script and fail to respect script boundaries 


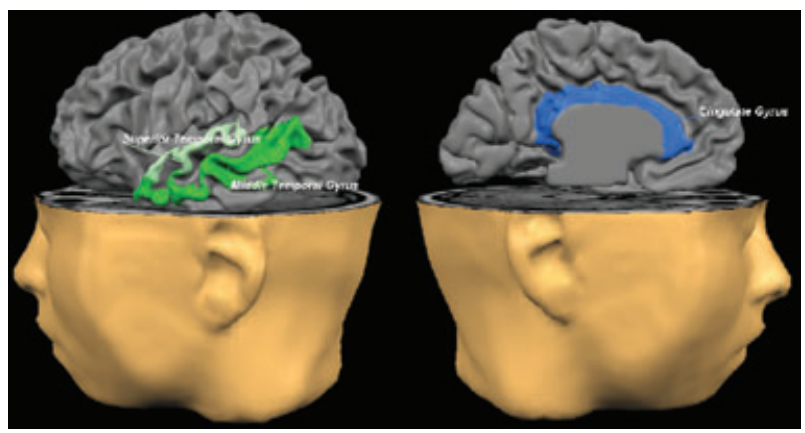

Figure 2 From left to right, the superior temporal gyrus, the middle temporal gyrus, and the cingulate gyrus.

(Sirigu, Zalla, Pillon, Grafman, Agid, et al., 1995; Sirigu, Zalla, Pillon, Grafman, Dubois, et al., 1995).

Comparative studies of prefrontal patients and patients affected by Parkinson's disease (for whom motor output via basal ganglia-thalamocortical pathways is inhibited $)^{1}$ reveal that although the prefrontal patients are impaired in the sequential ordering of events, and in respecting sequence boundaries and hierarchies, Parkinson patients fail to establish the contextual importance of each event within the planning activity (Zalla et al., 2000). A possible explanation is that the prefrontal cortex and the basal ganglia have different roles in action planning: During the generation of meaningful event sequences, the basal ganglia may, for example, serve to provide feedback about the value of the constituent action units (Zalla et al.). ${ }^{2}$

The multifunctionality of the prefrontal cortex in the temporal treatment of knowledge structures related to various knowledge domains arises from the particular internal subdivision of this brain area in specialized subparts (see Figure 1), each of which delimits the scope of a particular group of intersected functions (Wood \& Grafman, 2003). These functions include, for instance, domain-specific semantic working memory involved in the semantic analysis of initial occurrences of words and pictures, as well as in the selection of semantic alternatives (Gabrieli, Poldrack, \& Desmond, 1998).

According to Grafman (1995), knowledge is represented in terms of "structure event complexes" that are stored in distributed prefrontal subregions.

Recent monkey studies also outline the involvement of prefrontal neurons in processing goal-oriented behavioral sequences, reporting category-specific effects in the activity of prefrontal neuron subgroups. More specifically, during 
the planning of sequences of motor acts, such as paired action sequences (e.g., turn-turn-push-push), alternate action sequences (e.g., pull-turn-pull-turn), and four "repeat" sequences (e.g., turn-turn-turn-turn), cellular activity in the lateral prefrontal cortex appears to be selective for the specific category of the motor sequences to be performed rather than the sequence per se (Shima, Isoda, Mushiake, \& Tanji, 2007). As the category of the motor sequences to plan was not signaled by any cue but simply memorized during the training that preceded task execution, a neural representation of the category-related information specific to each type of motor sequence was internally generated within the lateral prefrontal cortex based on macrostructured action knowledge.

Neuropsychological and neuroimaging studies have explored the prefrontal functional organization with the purpose of detecting the domain dependency of the knowledge-related representations and computations. The findings have shed light on the existence of particular dissociations in the prefrontal regions, as will be outlined in the following sections.

\section{Prefrontal Parsing of Hierarchical Action Sequences}

Perceiving and understanding patterns of goal-directed action sequences, recognizing and attributing to them their relative and causal hierarchical order and goals within the action flow, presupposes the ability to locate events in time locations, by identifying the breakpoints that mark the transitions from one unit to the contiguous one (i.e., their initial and final boundaries).

Neuroimaging studies report, for example, that transient changes in the activity of a distributed network, including the right frontal cortex, are more sensitive to the segmentation of coarse event units than small ones (Zacks et al., 2001).

Neuropsychological work has examined the ability of brain-damaged patients with left or bilateral prefrontal lesions provoking deficits in action planning to determine the temporal margins of consecutive action sequences illustrated in video scenarios (Zalla et al., 2003). Action chunks located at different hierarchical levels, reflecting fine-grained and coarse-grained events, induce distinct detection strategies: Prefrontal patients exhibit a stronger deficit in segmenting large, higher order event units than small, low-level ones. This selective impairment of top-down chunking mechanisms, mirrored by a defective action monitoring and action planning, suggests that the prefrontal cortex contributes to both parsing and generation of meaningful goal-oriented action sequences. This indicates that the hierarchical organization of meaningful event units and subunits in the prefrontal regions influences the segmentation strategies of continuous, goal-oriented action streams. 
A recent hypothesis states that the hierarchical organization of plans, rather than the temporal organization of events, specifically drives the selection of actions in complex, goal-oriented behavior (Koechlin \& Jubault, 2006). The hypothesis is supported by neuroimaging data showing a phasic activation in the pars opercularis (BA 44) of the left inferior frontal gyrus when subjects process the boundaries of simple action chunks and in the pars triangularis (BA 45) for boundaries of hierarchically higher chunks of actions. According to this particular segregation of Broca's region (cf. Figure 1), a cascade model of brain activity associated to the nested levels of action hierarchies is proposed in which superordinate action chunks are processed by BA45, intermediate or simple action chunks are processed by BA44, and basic motor responses are processed by the premotor cortex.

The fact that these findings suggest the involvement of a brain region traditionally associated with language functions, such as Broca's area (BA 44, 45; Figure 1) (Broca, 1861), in the domain of action sequence, brings us back to reformulating the central question concerning the domain dependency of the sequential event knowledge representation and processing in the brain: Are there multiple cognitive mechanisms involved in sequential event structure processing related to different domains of knowledge? Are they controlled by distinct brain regions?

\section{Prefrontal Dissociations for Language Versus Action Sequences}

Whether the prefrontal cortex hosts independent mechanisms for different domains of temporally organized knowledge is a question explicitly addressed by lesion work comparing impairments in action planning and sequential structuring in syntax (Sirigu et al., 1998). In this study, sequence processing abilities were tested in 10 patients who exhibited selective lesions that included either part of the left inferior frontal gyrus (BA 44), and part of the left ventral premotor cortex (BA 6) (see Figure 3, left panel), or the left dorsolateral prefrontal cortex (BA 45, and part of BA 46) (see Figure 3, middle panel), or both (compounded lesion) (Figure 3, right panel).

The first group of patients showed impairments in tasks of planning and managing everyday activities. The second group of patients displayed agrammatic speech, with reduced syntactic abilities, impaired use of function words and grammatical endings, and generally poor, yet informative, verbal output. In the last group, both executive functions and language syntax were impaired. Two conditions were examined. In a syntax production condition, emphasizing morphosyntactic analysis, grammatically correct sentences had to be generated using 30 sets of cards. Each set contained the individual segments of one to four 


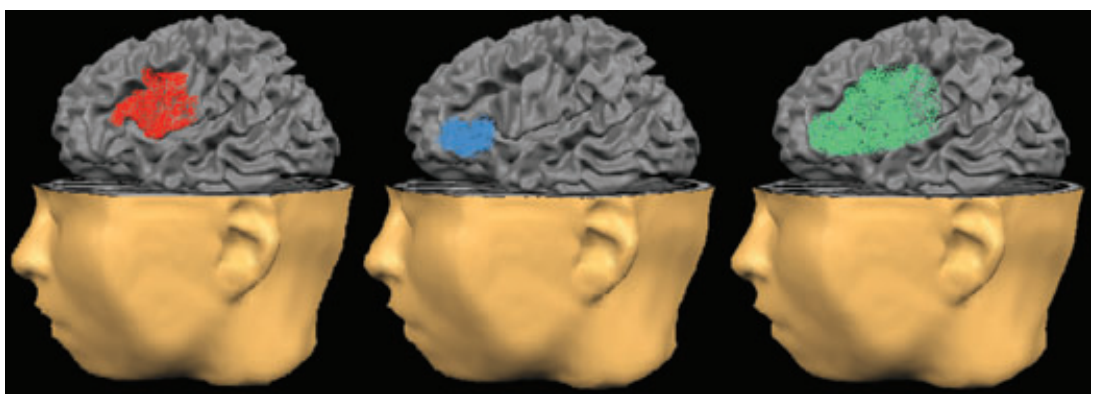

Figure 3 Location of the lesion areas common to each patient group. Left panel: Broca's lesion (BA 44); middle panel: prefrontal lesion (BA 45 and part of BA 46); right panel: compound lesion. (Adapted from Sirigu et al., 1998).

words, which served to form a complete sentence. In half of the sentences, the subject and the object were semantically reversible; that is, they could both play the semantic role of agent and patient, like "lady" and "man" in the example: "a lady was / pushed by / a man / while she / crossed the street" (from Sirigu et al., 1998, p. 773). This semantic reversibility was not present in the remaining half of sentences like in the example: "the butcher / sharpens his / knife / and cuts a / thick steak" (from Sirigu et al., 1998, p. 773). The reversibility condition was used in order to assess the patients' ability to process word order on the basis of purely syntactic rules, without semantic/contextual information. For instance, semantically reversible sentences prevented patients from adopting task-related strategies that can be available to some agrammatics, such as associating the animate items with the agent role and the subject position. Each sentence could be constructed in one correct order only based on morphosynctatic knowledge.

In a script condition, a correct action sequence had to be generated using a set of 20 cards, on each of which a single action was described using one to four words. Following the temporal sequence of actions depicted on the cards, a coherent short narrative had to be produced, for example: "arrive at the new stand / ask for the paper / take it / pay / leave" or "insert card / pick-up the receiver / dial / waiting for an answer / talk." Four different and semantically unrelated scripts could be composed using the actions depicted on the 20 cards, as there were five single actions for each script. The participants were required to establish the semantic content of the script by sorting out the sequences of cards that described a series of actions related to a same script. They then had to process the temporal structure of the script by attributing the appropriate temporal order to the sequence of actions selected for each script. The title of each possible script was indicated on a separate sheet. 
The generation of correct action sequences implied respecting temporal restriction rules constraining the action ordering. The task valorized the pragmatic analysis of the story grammar. The results of this study show that, in prefrontal patients, syntactic ordering was intact, whereas both the assignment of single actions to the correct script and the sequence between consecutive actions were severely altered. Broca's patients, in contrast, were able to accurately organize a coherent narrative but could not generate syntactically correct sentences. For instance, they made systematic inversions in the subject-object syntactic positions for the semantically reversible sentences illustrated so far (e.g., "man" was used instead of "lady"). Patients with compound lesions were disabled in both syntax and script abilities. The authors concluded that the double dissociation in the syntactic and script-related, pragmatic abilities is indicative of the involvement of different knowledge domains and mapped onto different networks within the prefrontal cortex (Sirigu et al., 1998). The results were consistent with previous findings showing that prefrontal patients are able to evoke the adequate action prototype (prototypicality of an action for a semantic category) associated with an action plan but impaired in the temporal ordering of actions (Sirigu, Zalla, Pillon, Grafman, Agid, et al., 1995). The fact that a lesion in Broca's area preserves the ability to temporally order words that refer to actions but not words referring to the sequential structure intrinsic to language indicates that sequence processing may be differently represented in the brain according to specific domains of knowledge.

Another result in this direction comes from a functional magnetic resonance imaging study investigating the perception of syntax compared to story grammar (i.e., the set of combinatory rules that specify the appropriate temporal constraints of an action sequence). The syntax and story grammar tasks required the perceptual detection respectively of either syntactic or action sequence errors in sentences visually projected on a screen. The results indicate that the perception of both syntax and story grammar recruits a partially overlapping network of regions (e.g., premotor areas, middle frontal gyrus, inferior frontal gyrus, anterior and posterior superior temporal sulcus, and supramarginal gyrus), more lateralizsed on the left hemisphere for syntax, more bilateral for the action sequences. The perception of story grammar, on the other hand, specifically activates the dorsolateral prefrontal cortex bilaterally, left supplementary motor area, and the left angular gyrus within the parietal cortex (Crozier et al., 1999). The bilateral prefrontal activation is interpreted as being particularly involved in event-sequence representations, temporal ordering of events, and action planning, within a broader fronto-parietal network specific to script processing. 
To summarize, globally, these data suggest a selective involvement of the prefrontal cortex in the perception of sequential relations between actions, and domain-specific organization for the representation of sequential event knowledge. However, a partial discrepancy must be noted between the data on the prefrontal dissociation for syntax and scripts, and the neuroimaging findings just presented, regarding the syntactic dissociation in Broca's area. Although a variable element can be found in the slightly different locations of lesions, and partly different tasks used, namely a generation and a perception task, respectively, it is useful to schematically relate these results to the perspective articulated around recent neurolinguistic insights, which in fact also reflect an at least double view of the role of Broca's area.

\section{What About Language Sequences?}

The selective involvement of Broca's pars opercularis (BA 44, cf. Figure 1) as reported by Sirigu et al. (1998), is consistent with the ample literature arguing for a specific role of the pars opercularis for syntax and pars triangularis (BA 45, cf. Figure 1) for semantics (e.g., thematic role processing) (Embick, Marantz, Miyashita, O’Niel, \& Sakai, 2000; Friederici, Wang, Herrmann, Maess, \& Oertel, 2000; see Moro et al., 2001, for an opposite view).

It is well known, however, that Broca's area, just as the prefrontal regions, exhibits an extremely multifunctional role in both linguistic and nonlinguistic domains. Without trying to disentangle this functional mosaic, we note that recent "extended" views propose a frontal nesting of regions in the left inferior frontal gyrus (with the inclusion of BA 44-45, BA 47, and BA 6), which appears to be functionally defined according to an anterior-ventral to posterior-dorsal gradient. Such embedded subregions get involved interactively in the integration of semantic, syntactic, and phonological information (Hagoort, 2005). For instance, PET results show that the Broca's pars opercularis (BA44) and the left Rolandic operculum, caudally adjacent to Broca's area, is recruited during syntactic encoding during speech production (Indefrey, Brown, et al., 2001), whereas the dorsolateral prefrontal cortex (BA 9), adjacent to Broca's area, is specifically involved in the processing of syntax rather than in the integration of syntactic and semantic information (Indefrey, Hagoort, Herzog, Seitz, \& Brown, 2001).

Recalling in part the cascade model proposed for the processing of action chunks (Koechlin \& Jubault, 2006), a nested model for the neural substrates of language processing suggests that the left inferior frontal gyrus is differentially involved in the computations aimed at the "unification" of different levels of linguistic material into more complex representations. Neuroimaging work 
showing an increase of neural activity in the posterior portion of Broca's area during the manipulation of temporal order of discrete units both linguistic (phonological) and nonlinguistic (vocal humming) (Gelfand \& Bookheimer, 2003), and Magnetoecephalography studies showing its involvement in the processing of musical syntax (Friederici, Rüschemeyer, Hahne, \& Fiebach, 2003) complement the insights, leading to the hypothesis that this area may have a multimodal role in higher order categorization and temporal processing that goes beyond the language domain.

\section{Implications and Future Inquiry}

This article has attempted to characterize the involvement of the prefrontal cortex in the mental operations that are required for the storage and retrieval of sequential event knowledge. It has focused on functional neuroimaging and lesion analyses of sequence processing of different types of knowledge domains, such as action and language.

Overall, the emerging picture from the studies reviewed here is that sequence processing may be modularly organized within the prefrontal cortex and in Broca's area, depending on the knowledge domain. In particular, the prefrontal cortex and Broca's areas play important but differential roles in the activation of the computational procedures necessary for the formation of sequential strings for action and language, respectively.

The proposal of multiple representational modes of sequential event structures may imply that the nature of the rules that specify the temporal order of sequential events covaries with the specific knowledge domain. For example, action sequences would be based on natural cause-effect and mean-ends relations. On the contrary, word order in language would follow an independent set of rules specific to language structure. The potential domain sensitivity of the computational operations that produce and process sequential event structures is compatible with dual-system models, which conceive of language as forming an independent and cognitive module, self-governed by combinatory rules unique to natural language. The data discussed so far provide evidence for a prefrontal dissociation between action and language sequences that is supportive of this view.

A clearer understanding of the nature of the computations performed in the prefrontal cortex and Broca's area, of their degree of abstraction and granularity, as well as of their potential pertinence for different knowledge domains will contribute to clarify this issue with respect to the more cognitive view of language, according to which grammar is a form of conceptualization and 
linguistic phenomena are thought to be stored and retrieved by the same cognitive resources that are required by the processing of knowledge structures belonging to nonlinguistic domains (Croft \& Cruse, 2004). Further exploration of the possible interplays between the neural underpinnings of action and language sequences may contribute to tease apart these perspectives, by testing whether the combinatory rules of language (the syntactic component) developmentally (and ontogenetically) depend on prelinguistic operations pertaining to the planning of simple motor action sequences (Steedman, 2002) or whether the rules are an autonomous set of procedural rules capturing regularities intrinsic to language (Pinker \& Ullmann, 2002).

From the neuroscientific point of view, the acquisition of novel spatial and temporal information about connectivity and real-time interactions of subareas within the prefrontal cortex with more distributed cerebral networks (e.g., neural projections and time-locked phase synchronizations of the neural activity in specific contiguous and noncontiguous brain subregions) will contribute to elucidate the temporal coding and processing of event sequences and to improve the current models of brain representations and activation of sequential event knowledge.

Further advances of cognitive neuroscience in this direction will open new perspectives for stimulating parallels and confrontation between the domains of language and action.

\section{Notes}

1 The subcortical structure of the basal ganglia typically intervenes in adaptative motor control (Mardsen, 1982), because the basal ganglia motor circuit modulates cortical output necessary for optimal movement. It has been pointed out that the basal ganglia are involved in more cognitive and motivational dimensions of behavior (Robbins \& Everitt, 1992), as well as in the retrieval, management, and constitution of goal-oriented action sequences (Graybiel, 1995).

2 Such a deficit may be caused by attentional set-shifting difficulties when the parallel manipulation of multiple types of information is demanded, rather than from impaired script event knowledge (Owen et al., 1993; Zalla et al., 1998).

\section{References}

Abelson, R. P. (1973). The structure of belief systems. In R. C. Schank \& K. M. Colby (Eds.), Computer models of thought and language (pp. 287-339). San Francisco: W. H. Freeman and Co. 
Bartlett, F. C. (1932). Remembering: A study in experimental and social psychology. Cambridge: Cambridge University Press.

Black, J. B., \& Bower, G. H. (1979). Episodes as chunks in narrative memory. Journal of Verbal Learning and Verbal Behavior, 18, 309-318.

Broca, P. (1861) Remarques sur le siège de la faculté du langage articulé, suivies d'une observation d'aphemie. Bulletin de la Société Anatomique de Paris, 36, $330-357$.

Chomsky, N. (1993). A minimalist program for linguistic theory. In K. Hale \& S. J. Keyser (Eds.), The view from building 20: Essays in linguistics in honor of Sylvain Bromberger (pp. 1-52). Cambridge, MA: MIT Press.

Chomsky, N. (1995). The minimalist program. Cambridge, MA: MIT Press.

Chomsky, N. (1998). Minimalist inquiries: The framework MITWPL. MIT Occasional Papers in Linguistics, 15, Department of Linguistics and Philosophy. Cambridge, MA: MIT.

Croft, W., \& Cruse, D. A. (2004). Cognitive linguistics. Cambridge: Cambridge University Press.

Crozier, S., Sirigu, A., Lehericy, S., Van de Moortele, P. F., Pillon, B., Grafman, J., et al. (1999). Distinct prefrontal activations in processing sequence at the sentence and script level: An fMRI study. Neuropsychologia, 37, 1469-1476.

Embick, D., Marantz, A., Miyashita, Y., O’Neil, W., \& Sakai, K. L. (2000). A syntactic specialization for Broca's area. Proceedings of the National Academy of Sciences USA, 97, 6150-6154.

Friederici, A. D., Wang, Y., Herrmann, C. S., Maess, B., \& Oertel, U. (2000). Localization of early syntactic processes in frontal and temporal cortical areas: A magnetoencephalographic study. Human Brain Mapping, 11, 1-11.

Friederici, A. D., Rüschemeyer, S. A., Hahne, A., \& Fiebach, C. J. (2003). The role of left inferior frontal and superior temporal cortex in sentence comprehension: Localizing syntactic and semantic processes. Cerebral Cortex, 13, 170-177.

Fuster, J. (1989). The prefrontal cortex. Anatomy, physiology, and neuropsychology of the frontal lobe. New York: Raven.

Gabrieli, J. D. E., Poldrack, R. A., \& Desmond, J. E. (1998). The role of the left prefrontal cortex in language and memory. Proceedings of the National Academy of Sciences, 95, 906-913.

Gelfand, J. R., \& Bookheimer, S. Y. (2003). Dissociating neural mechanisms of temporal sequencing and processing phonemes. Neuron, 38, 831-842.

Gobet, F., Lane, P. C. R., Croker, S., Cheng, P. C.-H., Jones, G., Oliver, I., et al. (2001). Chunking mechanisms in human learning. Trends in Cognitive Sciences, 5, 236-243.

Goldman-Rakic, P. S. (1987). Circuitry of primate prefrontal cortex and regulation of behavior by representational memory. In F. Plum (Ed.), Handbook of physiology, Section 1: The nervous system. Vol. 5: Higher functions of the brain (pp 373-417). Bethesda, MD: American Physiological Society. 
Grafman, J. (1989). Plans, actions, and mental sets: Managerial knowledge units in the frontal lobes. In E. Perecman (Ed.), Integrating theory and practice in clinical neuropsychology (pp. 93-138). Hillsdale, NJ: Erlbaum.

Grafman, J. (1995). Similarities and distinctions among current models of prefrontal cortical functions. In J. Grafman, K. J. Holyoak, \& F. Boller (Eds.), Structure and functions of the human prefrontal cortex (pp. 337-368). New York: New York Academy of Sciences.

Grafman, J. (1999). Experimental assessment of adult frontal lobe function. In B. L. Miller \& J. L. Cummings (Eds.), The human frontal lobes: Functions and disorders (pp. 321-344). New York: Guilford Press.

Graybiel, A. M. (1995). Building action repertoires: Memory and learning functions of the basal ganglia. Current Opinion in Neurobiology, 5, 733-741.

Grosz, B. J., \& Sidner, C. L. (1986). Attentions, intentions, and the structure of discourse. Computational Linguistics, 12(3), 175-204.

Hagoort, P. (2005). On Broca, brain, and binding: A new framework. Trends in Cognitive Sciences, 9, 416-423.

Indefrey, P., Brown, C. M., Hellwig, F., Amunts, K., Herzog, H., Seitz, R. J., et al. (2001). A neural correlate of syntactic encoding during speech production. Proceedings of the National Academy of Sciences USA, 98, 5933-5936.

Indefrey, P., Hagoort, P., Herzog, H., Seitz, R. J., \& Brown, C. M. (2001). Syntactic processing in left prefrontal cortex is independent of lexical leaning. Neuroimage, $14,546-555$.

Jackendoff, R. (2002). The mind doesn't work that way: The scope and limits of computational psychology. Language, 78, 164-170.

Koechlin, E., \& Jubault, T. (2006). Broca's area and the hierarchical organization of human behavior. Neuron, 50, 963-974.

Knutson, B., Westdorp, A., Kaiser, E., \& Hommer, D. (2000). FMRI visualization of brain activity during a monetary incentive delay task. Neuroimage, 12, 20-27.

Langacker, R. W. (1987). Foundations of cognitive grammar: Theoretical prerequisites (Vol. 1). Stanford, CA: Stanford University Press.

Levelt, W. J. M. (1989). Speaking: From intention to articulation. Cambridge, MA: MIT Press.

Mardsen, C. D. (1982). A mysterious motor function of the basal ganglia: The Robert Wartenberg lecture. Neurology, 32, 514-539.

Miller, G. A. (1956). The magical number seven, plus or minus two: Some limits on our capacity for processing information. Psychological Review, 63, 81-97.

Minsky, M. (1975). A framework for representing knowledge. In P. H. Winston (Ed.), The psychology of computer vision (pp. 211-277). New York: McGraw Hill.

Moro, A., Tettamanti, M., Perani, D., Donati, C., Cappa, S. F., \& Fazio, F. (2001). Syntax and the brain: Disentangling grammar by selective anomalies. Neuroimage, $13,110-118$. 
Owen, A. M., Roberts, A. C., Hodges, J. R., Summers, B. A., Polkey, C. E., \& Robbins, T. W. (1993). Contrasting mechanisms of attentional set-shifting in patients with frontal lobe damage or Parkinson's disease. Brain, 116, 1159-1175.

Partiot, A., Grafman, J., Sadato, N., Wachs, J., \& Hallett, M. (1995). Brain activation during the generation of non-emotional and emotional plans. Neuroreport, 6 , 1397-1400.

Partiot, A., Grafman, J., Sadato, N., Flitman, S., \& Wild, K. (1996). Brain activation during script event processing. Neuroreport, 7, 761-766.

Pinker, S. (1994). The language instinct. New York: William Morrow.

Pinker, S., \& Ullman, M. T. (2002). The past and future of the past tense. Trends in Cognitive Sciences, 6, 456-463.

Robbins, T. W., \& Everitt, B. J. (1992). Functions of dopamine in the dorsal and ventral striatum. Seminars in Neuroscience, 4, 119-127.

Schank, R. C. (1973). Identification of conceptualizations underlying natural language. In R. C. Schank \& K. M. Colby (Eds.), Computer models of thought and language (pp. 187-247). San Francisco: W. H. Freeman and Co.

Schank, R. C., \& Abelson, R. P. (1975). Scripts, plans, and knowledge. In Proceedings of the Fourth International Joint Conference on Artificial Intelligence, Tbilisi, Georgia, USSR (pp. 151-157). Cambridge, MA: Artificial Intelligence Lab.

Shallice, T. (1988). The allocation of processing resources: Higher-level control. From neuropsychology to mental structure (pp. 328-352). Cambridge: Cambridge University Press.

Shima, K., Isoda, M., Mushiake, H., \& Tanji, J. (2007). Categorization of behavioral sequences in the prefrontal cortex. Nature, 445, 315-318.

Simner, J., \& Pickering, M. (2004). Planning causes and consequences in discourse. Journal of Memory and Language, 52, 226-239.

Sirigu, A., Cohen, L., Zalla, T., Pradat-Diehl, P., Van Eeckhout, J., Grafman, J., et al. (1998). Distinct frontal regions for processing sentence syntax and story grammar. Cortex, 34, 771-778.

Sirigu, A., Zalla, T., Pillon, B., Grafman, J., Agid, Y., \& Dubois, B. (1995). Selective impairments in managerial knowledge following pre-frontal cortex damage. Cortex, 31, 301-316.

Sirigu, A., Zalla, T., Pillon, B., Grafman, J., Dubois, B., \& Agid, Y. (1995). Planning and script analysis following prefrontal lobe lesions. In J. Grafman, K. J. Holyoak, $\&$ F. Boller (Eds.), Structure and functions of the human prefrontal cortex (pp. 277-288). New York: New York Academy of Sciences.

Sirigu, A., Zalla, T., Pillon, B., Grafman, J., Agid, Y., \& Dubois, B. (1996). Encoding of sequence and boundaries of scripts following prefrontal lesions. Cortex, 32, 297-310.

Steedman, M. (2002). Plans, affordances, and combinatory grammar. Linguistics and Philosophy, 25, 723-752. 
Tannen, D. (1979). What's in a frame? Surface evidence for underlying expectations. In R. Freedle (Ed.), New directions in discourse processing (137-181). Norwood, NJ: Ablex.

Tannen, D., \& Wallat, C. (1987). Interactive frames and knowledge schemas in interaction: Examples from a medical examination/interview. Social Psychology Quarterly, 50, 205-216.

Ullman, M. T. (2001). A neurocognitive perspective on language: The declarative/procedural model. Nature Reviews Neuroscience, 2, 717-726.

Ullman, M. T. (2004). Contributions of memory circuits to language: The declarative/procedural model. Cognition, 92, 231-270.

Wood, A. G., Harvey, A. S., Wellard, R. M., Abbott, D. F., Anderson, V., Kean, M., et al. (2004). Language cortex activation in normal children. Neurology, 63, $1035-1044$.

Wood, J. N., \& Grafman, J. (2003). Human prefrontal cortex function: processing and representational perspectives. Nature Reviews Neuroscience, 4, 139-147.

Zacks, J. M., Tversky, B., \& Iyer, G. (2001). Perceiving, remembering, and communicating structure in events. Journal of Experimental Psychology: General, 130, 29-58.

Zalla, T., Sirigu, A., Pillon, B., Dubois, B., Grafman, J., \& Agid, Y. (1998). Deficit in evaluating pre-determined sequences of script events in patients with Parkinson's disease. Cortex, 34, 621-627.

Zalla, T., Koechlin, E., Pietrini, P., Basso, G., Aquino, P., Sirigu, A., et al. (2000). Differential amygdala responses to winning and losing: A functional magnetic resonance imaging study in humans. European Journal of Neuroscience, 12, $1764-1770$.

Zalla, T., Plassiart, C., Pillon, B., \& Sirigu, A. (2001). Action planning in a virtual context after prefrontal cortex damage. Neuropsychologia, 39, 759-770.

Zalla, T., Pradat-Diehl, P., \& Sirigu, A. (2003). Perception of action boundaries in patients with frontal lobe damage. Neuropsychologia, 41, 1619-1627. 